\title{
Primary Progressive Aphasias and Their Contribution to the Contemporary Knowledge About the Brain-Language Relationship
}

\author{
Michał Harciarek • Andrew Kertesz
}

Received: 22 June 2011 / Accepted: 25 July 2011 /Published online: 2 August 2011

(C) The Author(s) 2011. This article is published with open access at Springerlink.com

\begin{abstract}
Primary progressive aphasia (PPA), typically resulting from a neurodegenerative disease such as frontotemporal dementia/Pick Complex or Alzheimer's disease, is a heterogeneous clinical condition characterized by a progressive loss of specific language functions with initial sparing of other cognitive domains. Based on the constellation of symptoms, PPA has been classified into a nonfluent, semantic, or logopenic variant. This review of the literature aims to characterize the speech and language impairment, cognition, neuroimaging, pathology, genetics, and epidemiology associated with each of these variants. Some therapeutic recommendations, theoretical implications, and directions for future research have been also provided.
\end{abstract}

Keywords Progressive aphasia - Semantic dementia . Frontotemporal dementia $\cdot$ Pick Complex $\cdot$ Alzheimer's disease

\section{Acronym Key}

AD Alzheimer's disease

FTD frontotemporal dementia

CBD corticobasal degeneration

M. Harciarek ( $\square)$

Department of Social Sciences, Division of Clinical Psychology

and Neuropsychology, Institute of Psychology,

University of Gdańsk,

Bażyńskiego 4,

80-952 Gdańsk, Poland

e-mail: mharciarek@yahoo.com

A. Kertesz

Department of Neurology, University of Western Ontario,

London, ON, Canada

$\begin{array}{ll}\text { PSP } & \text { progressive supranuclear palsy } \\ \text { MND } & \text { motor neuron disease } \\ \text { SD } & \text { semantic dementia } \\ \text { PPA } & \text { primary progressive aphasia } \\ \text { LPA } & \text { logopenic progressive aphasia } \\ \text { PNFA } & \text { progressive non-fluent aphasia } \\ \text { PPA-G } & \text { primary progressive aphasia with } \\ & \text { agrammatism }\end{array}$

PPA-L logopenic primary progressive aphasia PPA-S semantic primary progressive aphasia nfvPPA non-fluent variant of primary progressive aphasia

lvPPA logopenic variant of primary progressive aphasia

svPPA semantic variant of primary progressive aphasia

bvFTD behavioral variant of frontotemporal dementia

ATAC argyrophilic, thorny astrocyte clusters

FTD-MND frontotemporal dementia, motor neuron disease type

MNDI motor neuron disease inclusions

FTLD-T frontotemporal lobar degeneration with tau

FTLD-U frontotemporal lobar degeneration with ubiquitin

MAPT microtubule-associated protein tau

PRGN progranulin

TDP-43 TAR DNA-binding protein 43

LRRK2 leucine-rich repeat kinase 2

FBI Frontal Behavioral Inventory

FDG-PET fluorodeoxyglucose positron emission tomography

HMPAO- hexamethylpropyleneamine oxime single

SPECT photon emission computer tomography 


\section{Introduction}

A progressive language disturbance resulting from circumscribed atrophy of the left hemisphere was first described in 1892 by Arnold Pick, who reported a 71-year-old patient with a 2-year history of gradually progressive symptoms resembling transcortical sensory aphasia (Pick 1892). A few years later, Pick presented a similar case he called primary progressive dementia with left temporal atrophy, and classified the aphasia as "pure word deafness" (Pick 1904). Practically at the same time, several other cases of language disorder due to frontotemporal atrophy were described, confirming Pick's initial observations that a single, circumscribed atrophic process may result in progressive aphasia (Dejerine and Serieux 1897; Rosenfeld 1909; Serieux 1893). In some of these cases, language impairment was an initial and a relatively isolated sign of a neurodegenerative condition, predominantly Pick's disease (e.g., Rosenfeld 1909). Moreover, Caron (1934), in his review of Pick's disease, stated that its most common form is characterized by early development of aphasia. It is worth mentioning that Alzheimer's first patient was also aphasic (Alzheimer 1907), suggesting that language impairment may also be a component of Alzheimer's disease (AD). Nonetheless, aphasia as a prominent feature of AD has been emphasized only recently.

For most of the $20^{\text {th }}$ century, however, aphasia as a result of a circumscribed atrophic process was a relatively neglected phenomenon, until Mesulam presented a series of cases with "slowly progressive aphasia," subsequently renamed "primary progressive aphasia" (PPA) (Mesulam 1982, 1987, 2001). He also proposed to differentiate this syndrome from AD. Others at the same time described cases of aphasia with autopsy-proven Pick's disease (Wechsler 1977; Holland et al. 1985). Importantly, Mesulam's patients were severely anomic, and most of them had impaired speech fluency accompanied by frequent grammatical errors. This form of progressive language disorder was therefore later renamed "progressive nonfluent aphasia" (PNFA) (Grossman et al. 1996), and more recently "primary progressive aphasia with agrammatism" (PPA-G) (Mesulam et al. 2009). The condition was considered a separate entity for a while, but evidence was presented to include it under the umbrella of frontotemporal dementia (FTD)/Pick complex (Kertesz et al. 1994).

In mid 70's, Warrington described three patients with progressive loss of meaning on naming, and of word and picture comprehension in the absence of impaired syntax and phonology. Moreover, their perceptual and visuospatial abilities as well as their day-to-day memory were preserved. Warrington suggested that her patients exhibited selective breakdown of semantic memory (Warrington 1975). This condition, being somewhat reminiscent of one of Pick's cases, was subsequently described by others and first lumped with PPA (e.g., Basso 1988; Mehler et al. 1986; Poeck and Luzzatti 1988; Schwartz et al. 1979) and later named "semantic dementia" (SD) (Snowden et al. 1989). Later, Hodges and colleagues (1992) provided a comprehensive characterization of SD.

Since early 1990's, individuals with PPA have been classified as SD vs PNFA, or in some studies as "fluent" vs "nonfluent" (see Harciarek and Jodzio 2005), analogous to this basic distinction in stroke aphasia. Nonetheless, the fluency-nonfluency dichotomy in PPA is very problematic, mainly because there is no accepted definition of fluency, despite several attempts at quantitation (Ash et al. 2010; Kertesz et al. 2003, Knibb et al. 2009; Mendez et al. 2003; Wilson et al. 2010). Moreover, speech fluency is a dynamic and multidimensional feature of language depending on several premorbid output characteristics (e.g., speech rate, articulary ability) as well as the stage of the disease (Kertesz et al. 2003). Thus, given that there were many patients with mixed PPA who did not fit the binary classification, attempts have been made to fill this gap between the PNFA and SD. As a result, Gorno-Tempini and her colleagues $(2004,2008)$ have recently identified a third clinical variant -logopenic progressive aphasia (LPA). Logopenic aphasia, with hesitant anomic speech, word retrieval and sentence repetition deficits among its core features, is a relatively common variant of PPA, but more than half of the cases turn out to be AD. By comparison, PNFA is a better predictor of a tauopathy, and SD is associated with ubiquitinated inclusions (see below).

Overall, PPA is a clinical condition characterized by a gradual, progressive loss of specific language functions, with relative sparing of other cognitive domains as well as activities of daily living over approximately the first 2 years of the disease (Gorno-Tempini et al. 2011; Mesulam 2001, 2003). The progressive language impairment, resulting from a neurodegenerative disease such as FTD/Pick Complex or $\mathrm{AD}$ (see Figure 1), includes language production, object naming, syntax or word comprehension. Based on specific symptoms or performance of these functions, PPA can be classified into one of the three variants: nonfluent, logopenic, or semantic. Each syndrome seems to have a different distribution of atrophy (Gorno-Tempini et al. 2004), and is associated with different likelihood of particular underlying pathology (Davies et al. 2005; Josephs et al. 2006, 2008; Knibb et al. 2006; Mesulam et al. 2008). Hence, by presenting the clinical, neuropsychological, neuroimaging, pathological, and genetic features, this review aims to comprehensively characterize each of these three variants in order to better understand their courses as well as their underlying neurodegenerative processes. Some therapeutic recommendations, theoretical implications, and directions for future research have been also provided. 


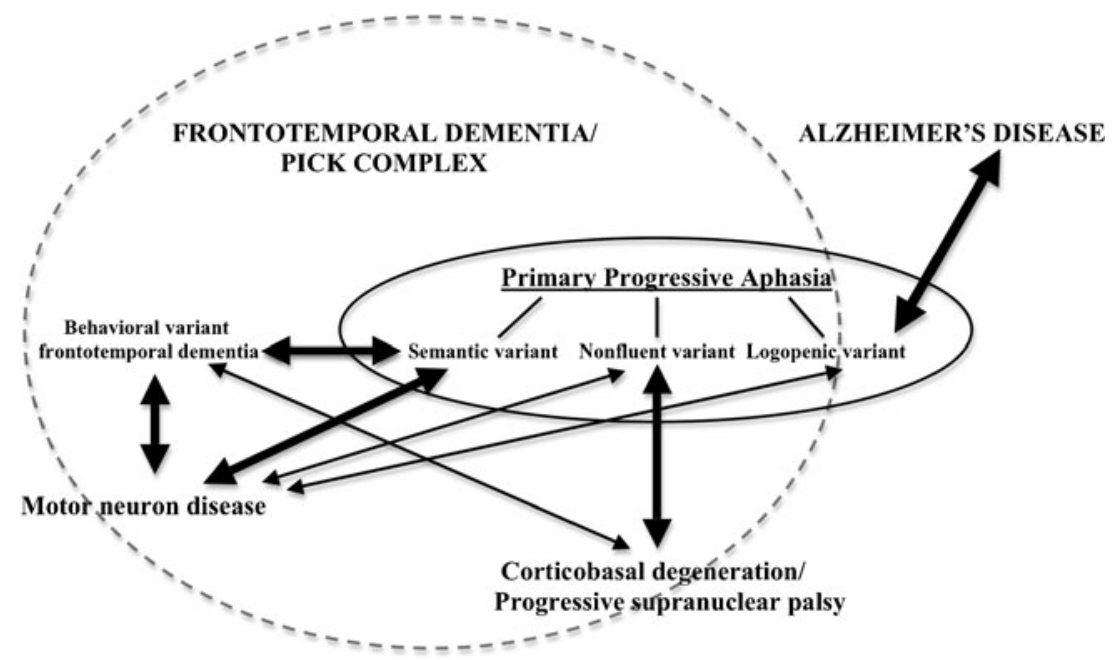

Fig. 1 The place of primary progressive aphasia among neurodegenerative diseases. Note: The thickness of arrows represents the approximated extent of the overlap (association) between specific syndromes

\section{Nonfluent Variant PPA}

\section{Clinical Features}

The first symptoms of the nonfluent variant PPA (nfvPPA), also known as PNFA (Grossman et al. 1996) or PPA-G (Mesulam et al. 2009), might be heterogeneous, but initially almost all individuals present with increasing anomia as well as word finding difficulties (Ash et al. 2010; Blair et al. 2007; Clark et al. 2005; Harciarek and Kertesz 2009; Kertesz et al. 2003; Knibb et al. 2009; see also Selnes and Harciarek 2006). In this respect, these patients are not much different from aphasics with $\mathrm{AD}$, except they have relatively preserved memory and non-verbal cognition (Blair et al. 2007; Mesulam et al. 2008; Weintraub et al. 1990). The speech fluency is then still relatively preserved, although some patients start having problems with articulation and, thus, their output begins to be effortful and halting. As the disease progresses, the language becomes more impaired and the speech fluency decreases. Nonetheless, even mildly-affected subjects with nfvPPA frequently experience progressive problems with sentence construction and syntax so that their speech becomes agrammatic and difficult to understand (Gunawardena et al. 2010; Kertesz 2008; Mesulam et al. 2009; Rohrer et al. 2010a; Weintraub et al. 1990; Turner et al. 1996). The patients' conversational speech is characterized by a significant shortage of verbs (Hillis et al. 2002, 2004), although this effect seems to be modified by word frequency. Also, subjects with nfvPPA often omit required determiners, and fail to produce appropriate subject-verb agreement (Ash et al. 2009). Additionally, these individuals may have impaired motor planning and sequencing of the movements required for correct speech production, regarded as an apraxia of speech (Gorno-Tempini et al. 2004; Josephs et al. 2006; Kertesz et al. 2003). This may be one of the initial features of nfvPPA, and these subjects are less likely to be mistaken for AD.

Although agrammatism is now among the core features of nfvPPA (Gorno-Tempini et al. 2011), and the speech output of some of subjects may indeed resemble a traditional Broca's aphasia (Blair et al. 2007), the agrammatism in nfvPPA is typically less severe than that seen after stroke (Clark et al. 2005; Graham et al. 2004; Kertesz et al. 2003; Knibb et al. 2009; Patterson et al. 2006). Nonetheless, because not only repetition of longer sentences and multisyllabic words (e.g., hippopotamus, tachistoscope) (Hodges et al. 2008) but also of relatively short words and phrases is frequently impaired, many patients with nfvPPA may be classified as having conduction aphasia characterized by poor repetition but intact auditory comprehension and fluent yet paraphasic speech (Blair et al. 2007). A vast subset of patients, especially those with apraxia of speech, may struggle with the first consonants of a word, make pauses in mid-word, commit phonological errors, as well as have a slow rate, reduced complexity, and defective prosody of speech (Budd et al. 2010; Wilson et al. 2010). In addition, they often have severe difficulties repeating strings of syllables, particularly those requiring the ability to properly coordinate complex articulatory movements (e.g., "pa, ta, ka, ....pa, ta, ka") (Ogar et al. 2007). Of note, however, is a recent study by Rohrer and coworkers (2010a) that suggests that patients with apraxia of speech may have relatively better confrontational naming than those without speech apraxia. Occasionally, individuals have dysarthria with systematic distortion of speech that mirrors articulatory problems seen in motor neuron disease (MND) (Duffy et al. 2007; Soliveri et al. 2003). In such 
cases, written production tests (such as a written description of a picture) or syntax comprehension tasks are useful because they can reveal even mild grammatical errors in nfvPPA (Weintraub et al. 1990, 2009).

Nevertheless, there is an ongoing dispute whether language difficulties in nfvPPA are mainly driven by motor versus nonmotor language impairment. This question has been recently addressed by Ash and coworkers (2010) who found that the majority of speech sound errors $(82 \%)$ were substitutions, insertions, deletions, or transpositions, all of which contain actual features of American English speech and likely represent incorrect retrieval of the desired sound. Interestingly, only $18 \%$ of errors represented a motor impairment. Moreover, in agreement with other findings (Knibb et al. 2009), no association was observed between the type of error and the speech rate or syntactic complexity, but a correlation was seen with grammatical correctness. Thus, these findings suggest that apraxia of speech may not be the central cause of the slowed, effortful speech that characterizes nfvPPA. Rather, nonmotor language impairment, agrammatism, combined with phonological retrieval is likely to contribute more to this dysfluency.

As a point of comparison, semantic deficits are not seen in nfvPPA (Kertesz et al. 2005, 2007), and the occasional presence of semantic errors seems to be predominantly related to the impaired articulatory planning and programming involved in word production (Budd et al. 2010). Moreover, even at the end-stage of this condition, characterized by complete mutism, patients have relative preservation of comprehension, unlike in global aphasia, $\mathrm{SD}$ or in severe AD (Appell et al. 1982; Karbe et al. 1993; Kertesz et al. 2010; Weintraub et al. 1990). In subjects with agrammatism, however, comprehension of sentences may be impaired, especially those with complex syntactic constructions, such as negative passives and object relative clauses (e.g., "The picture was not given by a girl") (GornoTempini et al. 2004; Grossman et al. 1996; Hodges and Patterson 1996; Peelle et al. 2008; Rohrer et al. 2010a; see also Grossman and Ash 2004). For the same reason, patients with nfvPPA may also fail on understanding sequential commands (Blair et al. 2007), particularly multi-part commands. Single-word comprehension and object knowledge are, however, well preserved (Harciarek and Kertesz 2009; Hodges et al. 2008; Koenig et al. 2006; Libon et al. 2007). As the aphasia progresses, many individuals exhibit reading difficulties, although these problems are typically mild and phonemic in nature (Patterson et al. 2006; Rohrer et al. 2010a). By comparison, writing is often markedly impaired, with a vast number of grammatical errors (Graham et al. 2004).

According to the operational criteria by Mesulam (2001, 2003), as well as to the most recent classification by Gorno-
Tempini and colleagues (2011), PPA is characterized by a relative preservation of nonverbal cognition in the first 2 years of the illness. Importantly, however, many cases with the nfvPPA have extrapyramidal features of corticobasal degeneration (CBD) and progressive supranuclear palsy (PSP), which appear before the 2 years deadline (Kertesz et al. 2000, 2005, 2007; Rohrer et al. 2010b). In some cases early, progressive limb apraxia can be a prominent feature (Fukui et al. 1996; Rohrer et al. 2010c), indicating further clinical overlap between nfvPPA and CBD/PSP (Kertesz et al. 2010; McMonagle et al. 2006). Some of these patients develop early behavioral features characteristic for the behavioral variant FTD (bvFTD). For example, Marczinski and coworkers (2004), using the "Frontal Behavior Inventory" (FBI) (Kertesz et al. 1997, 2000b), followed behavioral changes in nfvPPA over a3 year period. They found that, in addition to early apathy symptoms (see also Rohrer and Warren 2010), there was a significant increase in disorganization, inattention, poor judgment, inappropriateness, aggression, and hyperorality. Moreover, by the third year of testing, FBI scores of patients with nfvPPA were approaching scores of patients with bvFTD, further supporting the clinical overlap between the variations of this dementia (see Kertesz et al. 1994, 2005, 2007). Also, some patients may develop early executive and working memory problems (Patterson et al. 2006), although these abnormalities may not impact subjects' day-to-day functioning.

\section{Neuroimaging}

The majority of patients with nfvPPA present with structural and metabolic changes restricted to their dominant language hemisphere, typically in the area of the left sylvian fissure (Caselli and Jack 1992; GornoTempini et al. 2004, 2008; Mesulam 2003; Mesulam et al. 2008; Sonty et al. 2007; Wilson et al. 2009a). More specifically, abnormalities in the left posterior frontoinsular region, i.e. inferior frontal gyrus (Broca area), insula, premotor and supplementary motor areas have been usually detected in patients with apraxia of speech and agrammatism (Hu et al. 2010; Mesulam et al. 2009; Nestor et al. 2003; Rabinovici et al. 2008; Rohrer et al. 2010d; Whitwell et al. 2010). Nonetheless, some individuals with this variant have been shown to have more generalized atrophy (Cappa et al. 1996), and there is a subset of cases with unremarkable imaging (Mesulam et al. 2008). Thus, neuroimaging techniques in the setting of nfvPPA, although helpful throughout the diagnostic process, have been predominantly important to exclude nonneurodegenerative causes of aphasia such as cerebrovascular disease or tumor. 
Neuropathology

Similarly to both clinical and neuroimaging findings, there is no one clear neuropathological picture associated with nfvPPA. Nevertheless, the histopathological assessment in most cases reveals Pick's disease or CBD with neurons containing a microtubule-associated protein tau (MAPT) (Davies and Xuereb 2007; Josephs et al. 2006; Kertesz et al. 1994; Kertesz et al. 2005; Knibb et al. 2006; Mesulam et al. 2008). For example, Mesulam and coworkers (2008) have recently subdivided progressive nonfluent aphasia according to Neary et al. criteria (1998) into agrammatic and logopenic variants, as Gorno-Tempini and colleagues (2004) proposed. In their sample of 23 autopsies, all 6 agrammatic cases had frontotemporal lobar degeneration associated with tau (FTLD-T). The literature, however, describes a few nonfluent cases with AD pathology (plaques and tangles) (Greene et al. 1996) or nfvPPA cases with ubiquitinated frontotemporal lobar degeneration [FTLD-U; also known as frontotemporal dementia, motor neuron disease type (FTD-MND) or motor neuron disease inclusions (MNDI)] characteristic for MND (Kertesz et al. 2005, Snowden et al. 2006, 2007). Subjects with FTLD-U may additionally have co-localized antibodies to abnormal TAR DNA-binding protein 43 (TDP-43) that have been recently demonstrated to be more prominent in the language areas of these cases (Gliebus et al. 2010). Sometimes nfvPPA has been also associated with clear CBD (astrocytic plaques), or PSP (tufted astrocytes) pathology (Josephs et al. 2005; Kertesz et al. 2005), indicating further overlap with these syndromes.

\section{Genetics}

From all patients with nfvPPA, only a subset has a family history of this syndrome. Some of these cases may present with mutations in MAPT gene (Munoz et al. 2007a; Pickering-Brown et al. 2008), and some may have mutations in the progranulin (PGRN) gene (Benussi et al. 2009; Mesulam et al. 2007; Pickering-Brown et al. 2008; Rohrer et al. 2008; Snowden et al. 2006, 2007). Patients with the PRGN mutations may have a later onset of the disease, and more frequently present with various types of apraxia (Pickering-Brown et al. 2008). Interestingly, nfvPPA may be also associated with mutations in the leucine-rich repeat kinase 2 (LRRK2) gene that have been typically seen in both familial and sporadic parkinsonism (Chen-Plotkin et al. 2008).

Epidemiology and Treatment

Apart from cases associated with MND, the progression of nfvPPA is fairly slow, with an average survival from about
8 to 10 years (Hodges et al. 2003; Kertesz et al. 2005, 2007). The prevalence of this variant seems to be equal in men and women and, so far, there are no other risk factors but age (Clark et al. 2005; Westbury and Bub 1997), with first symptoms between the age of 60-65. Nonetheless, an increased incidence of nfvPPA has been recently demonstrated in men who had undergone vasectomy (Weintraub et al. 2006). A link between vasectomy and PPA was postulated to be a response generated after the blood-testis barrier is broken and an autoantigen, such as tau, expressed on spermatozoa, is exposed to the immune system. Of note, however, Han et al. (2010) did not find any association between antisperm antibodies and language dysfunction in $\mathrm{AD}$, also a tauopathy.

Although no reliable therapy for nfvPPA yet exists, a few medications as well as some non-medication approaches, such as speech therapy, have been tried. For example, a recent trial of galantamine (a cholinesterase inhibitor) in bvFTD and PPA found a trend for benefit in language function and global severity in the nfvPPA subgroup (Kertesz et al. 2008). Also, Boxer and coworkers (2009) have shown that memantine is a well-tolerated drug in patients with FTD, which may slow down the progression of cognitive decline in nfvPPA. Nonetheless, treatment of nfvPPA remains predominantly symptomatic, especially with regard to behavioral disturbances such as obsessions, restlessness, and roaming. In these cases, selective serotonin reuptake inhibitors such as trazodone may be most effective (Lebert et al. 2004; Swartz et al. 1997).

\section{Semantic Variant PPA}

\section{Clinical Features}

Semantic variant PPA (svPPA), originally named semantic dementia (SD) by Snowden et al. (1989), also called "primary progressive semantic aphasia" (Kertesz et al. 1998), "fluent PPA“ (Adlam et al. 2006; Clark et al. 2005), "temporal variant FTD“ (Bozeat et al. 2000a), "semantic PPA" (PPA-S) (Mesulam et al. 2009), or, in Japanese literature "Gogi (word meaning) aphasia" (Tanabe et al. 1992), is the second most extensively described variety of progressive aphasia associated with FTD/Pick disease after nfvPPA. This condition has been usually characterized by a progressive and multimodal loss of semantic knowledge (Hodges et al. 1992; Julien et al. 2008; Kashibayashi et al., 2010; Mayberry et al., 2010; Snowden et al., 1989). Thus, most current descriptions have adopted the term "SD" (see Hodges and Patterson 2007). Nonetheless, these patients are clinically more aphasic than demented, and the primary semantic impairment is most 
often seen in a language domain, particularly in naming and comprehension (Adlam et al. 2006; Hodges et al. 1992; Kertesz et al. 1998, 2010; Knels and Danek 2010; Libon et al. 2009; Mesulam et al. 2009). Thus, these typically early cases of SD may be referred to preferentially as SvPPA (see Gorno-Tempini et al. 2011). These patients progressively lose the meaning of words, but are fluent and repeat well. In this respect, svPPA seems to be similar to "transcortical sensory aphasia" in which articulation, repetition, phonology, and syntax are preserved but the patient has poor comprehension as well as severe anomia and word finding difficulty. In fact, transcortical sensory aphasia has been long recognized as a clinical pattern with degenerative pathology (Henschen 1922). Initially, patients produce circumlocutions, but the rate of their speech is normal. Nonetheless, patients often overuse closed-class words, pronouns and verbs, and higher frequency nouns, reflecting lexical retrieval deficits (Bird et al. 2000; Meteyard and Patterson 2009; Wilson et al. 2010). As the disease progresses, their speech is still considered fluent, but characterized by semantic jargon, frequently unrelated to the questions being asked or the topic discussed (Kertesz et al. 1998, 2010). Also, as a result of their lexical-semantic impairment, the length of patients' connected speech becomes increasingly shorter, eventually leading to mutism (op.cit.).

Severe, progressive anomia and markedly impaired comprehension of single words with patients themselves frequently asking the meaning of words, usually nouns, are the hallmark of svPPA (Blair et al. 2007; Gorno-Tempini et al. 2004, 2011; Harciarek and Kertesz 2009; Hodges et al. 1992, Hodges et al. 2008; Kertesz et al. 1998, 2010; Snowden et al. 1989; see also Mesulam 2003). Importantly, defective naming and single-word comprehension is especially strong for low familiarity items during the earliest stages (e.g., 'porcupine' vs the more familiar/frequent 'dog'), which may be the only sign of the disease at this point (Caine et al. 2009; see also Hodges and Patterson 2007). Later, these problems also become evident for more typical words:

Caregiver: "Could you pass me the knife please?"

Patient: "Knife? .... knife .... what is knife?".

Of note, we found that the "What is...?" question was the primary diagnostic feature of svPPA in our recent study (Kertesz et al. 2010). In spontaneous speech and on specific language testing, less frequent words are substituted with more familiar ones, typically from a superordinate category: 'animal' for 'dog', 'thing' for 'fork'. Most patients produce semantic paraphasias, especially during object naming. Severe difficulties defining words are also characteristic of individuals with svPPA, who often provide only general or inaccurate definitions. Due to well-preserved phonology, subjects with svPPA can correctly repeat names, even a multisyllabic word such as 'hippopotamus,' similar to that seen in transcortical sensory aphasia (Hodges et al. 2008).

Another striking and relatively early feature of this clinical condition is the profound pragmatic disturbance that can be commonly observed both during a conversational speech and upon a neuropsychological assessment (e.g., during defining words or a picture/scene description) (Ash et al. 2006; Kertesz et al. 2010). The majority of patients with svPPA present with garrulous, excessive, and frequently disinhibited output that is characterized by stereotypic thematic perseverations and not stopping to listen. This conversational peculiarity appears early, although it is often difficult to define by the casual observer. Most caregivers will say "He never listens, just carrying on and on with his own topic". Eventually, severe pragmatic impairment, together with frequent questioning the meaning of words, significantly contributes to the social and communication handicap.

A widespread semantic memory deficit leads to defective object recognition, also when presented from other sensory modalities such as vision, tactility, olfaction, and gustation (Adlam et al. 2006; Bozeat et al. 2000b; Hodges et al. 1992; Luzzi et al. 2007; Snowden et al. 1989). Thus, as the disease progresses, the early lexical-semantic problems seem to be accompanied by different forms of agnosia (e.g., visual agnosia; Kertesz et al. 1998, 2010). The ability to correctly identify objects is strongly modified by factors such as the type of material (real object vs. pictorial representations), as well as object familiarity and typicality (Mayberry et al. 2010; see also Hodges and Patterson 2007). For example, patients may not have problems identifying common objects such as a spoon or key, whereas the recognition of less frequently used tools (e.g., a saw or stethoscope) would be markedly impaired. Moreover, given that an amodal semantic representation has been suggested to be degraded in svPPA, semantic generalization is also very limited (Lambon Ralph and Patterson 2008). For example, if a knife used at home is made of silver, a white plastic knife in a testing room may not be correctly identified. Furthermore, some patients may exhibit a severe impairment in the recognition of faces, known as progressive prosopagnosia (Josephs et al. 2008; Thompson et al. 2003). Importantly, this specific form of visual agnosia is also familiarity-dependent, although faces of well-known family members eventually become poorly recognized as well (Mondini and Semenza 2006).

Given that patients with svPPA have preserved phonology, they can still correctly relate letters to sounds while reading regular words. Nonetheless, similar to meaning, the sound of an irregular word is not related to its spelling, and must be learnt separately for each word. Hence, because 
these patients progressively lose the meaning of words, they are no longer able to read by meaning. Instead, they start reading only by phonology. As a result, irregular words are pronounced as if they were regular (e.g., 'yatst' for "yacht", 'dufnut' for 'doughnut'), a phenomenon widely recognized as surface dyslexia (Fushimi et al. 2009; Jefferies et al. 2004; Kertesz et al. 1998, 2010; Wilson et al. 2009b). This also applies to writing (surface dysgraphia), because some patients may additionally have impaired spelling (Caine et al. 2009).

Interestingly, despite a multimodal semantic loss for most categories, some lexical-semantic categories in patients with svPPA have been demonstrated to be recognized better than others (for review see LambonRalph et al. 2003). For example, some individuals may have more difficulty processing the meaning of nouns rather than verbs (Daniele et al. 1994; Hillis et al. 2004; Kertesz et al. 1998), although a reverse dissociation has been also observed (Yi et al., 2007). Additionally, subjects with svPPA appear to have disproportionate difficulty understanding concrete concepts compared to abstract concepts (Breedin et al. 1994; Kertesz et al. 1998; Weinstein et al. 2011). Furthermore, cases have been described with greater, or even selective, deficits for people and animals (see Gainotti 2007). In contrast to patients' defective processing of names of objects, geometric figures, and body parts, their numerical and color knowledge seems to be relatively spared (Cappelletti et al. 2001; Crutch and Warrington 2002; Halpern et al. 2004; Harciarek and Kertesz 2009; Robinson and Cipolotti 2001; Zamarian et al. 2006). Nonetheless, it has been recently observed that reverse imageability effects (i.e., relative preservation of abstract knowledge) is not a characteristic feature of svPPA (Hoffman and Lambon Ralph 2011; Jefferies et al. 2009). Moreover, Julien and colleagues $(2008,2010)$ have shown that conceptual understanding of arithmetic may also degrade progressively.

Semantic variant is strongly associated with bvFTD, and so early behavioral and personality changes are almost always seen in patients with this type of PPA (Edwards-Lee et al. 1997; Kertesz et al. 2007, 2010; Neary et al. 1998; Rosen et al. 2006; Seeley et al. 2005; Snowden et al. 1989). These abnormalities typically include a combination of disinhibition, irritability, increased social seeking, and food fads such as the development of a sweet tooth. The peculiar, bizarre food choices have been even suggested to be a characteristic feature of svPPA (Snowden et al. 2001). Additionally, many subjects develop a strong interest in new religious movements as well as start to dress in a very eccentric fashion (Edwards-Lee et al. 1997). Lack of empathy, utilization behaviors, mental inflexibility, and compulsions - predominantly clockwatching and intense interest in jigsaws - are also frequently noted, although they are more likely to appear at the later stages of the disease (Seeley et al. 2005). Interestingly, in comparison to individuals with nfvPPA, patients with svPPA are typically unaware of their progressive language impairment (see Kertesz 2010).

In contrast to the early semantic deficits and behavioral abnormalities, episodic memory, particularly for autobiographical events, seems to be preserved in svPPA (Graham et al. 2000; Hodges and Graham 2001; Kertesz et al. 1998; Scahill et al. 2005). Moreover, patients with svPPA have excellent recognition memory, although their recognition relies predominantly on perceptual rather than on their degraded semantic system. Thus, they may correctly learn and recognize even a degraded item but only if it is perceptually identical with the 'to be remembered' stimulus (Graham et al. 2000; Simons et al. 2001). As the disease progresses, however, episodic memory may also become impaired (Matuszewski et al. 2009). When these deficits emerge, they have been shown to have a unique pattern, with patients having difficulty recalling remote but not dayto-day memories (a reverse of the Ribot's law) (Nestor et al. 2002; see also Harciarek and Jodzio 2005). Also, patients with svPPA do not present with impaired orientation to time and place (Hodges et al. 1992). Additionally, their perceptual, spatial, and motor abilities are typically very wellpreserved, so that they often can copy or paint colorful pictures and drawings (see Kertesz 2006). In contrast to cases with nfvPPA, they can easily solve relatively complex jigsaw puzzles (Green and Patterson 2009). Nonetheless, when asked what is in the picture they painted or the puzzle they solved, patients with svPPA often reveal no knowledge about the meaning of these objects; they frequently say "I don't know" or just vaguely describe what they see (e.g., "it's running" for a painted scene of a dog standing next to the house).

\section{Neuroimaging}

Neuroimaging of the svPPA has provided the most consistent picture of the anatomy of the neurodegenerative process across all three variants of PPA. Even early in its course, svPPA has been almost always associated with bilateral atrophy and decreased metabolism in the anterior temporal lobes, typically greater on the left (Diehl et al. 2004; Hodges et al. 1992; Galton et al. 2001; GornoTempini et al. 2004; Mummery et al. 2000; Rabinovici et al. 2008; Rosen et al. 2002). As the disease progresses, the atrophic process begins to involve the ventral and lateral parts of temporal lobes, including the fusiform gyrus. This focal anatomical involvement in svPPA is typically quite prominent, making neuroimaging a very useful feature in the diagnostic process of this PPA variant.

Lexical-semantic impairment has also been shown to be associated with greater left-sided atrophy, whereas a 
degradation of knowledge about people, including progressive prosopagnosia, as well as pronounced behavioral changes have been predominantly seen in cases with more extensive temporal atrophy in the right hemisphere (Chan et al. 2009; Davies et al. 2005; Josephs et al. 2008; Thompson et al. 2003). Also, the hippocampi are often well preserved in most cases (Chan et al. 2001), explaining why patients with svPPA have typically well-preserved episodic memory.

\section{Neuropathology}

In comparison to other forms of PPA, the neuropathological changes in svPPA seem to be relatively homogeneous, with the majority of cases having a morphological picture characteristic of FTLD-U pathology with ubiquitinpositive, tau-negative inclusions (Davies et al. 2005; Kertesz et al. 2005). Rare cases, however, may present with Pick bodies and Pick cells as well as with AD pathology, especially subjects who subesquently developed episodic memory deficits (see Hodges and Patterson 2007).

\section{Genetics}

Many of the familial svPPA cases have mutations in the PGRN and some in the MAPT gene (Pickering-Brown et al. 2008). More recently, a case of a 46-year-old woman suffering from progressive anomia and prosopagnosia was described (Bessi et al. 2010). Genetic testing revealed a missense mutation V363I in exon 12 of the MAPT gene.

\section{Epidemiology and Treatment}

Although detailed epidemiological data regarding svPPA are still lacking, Chow and coworkers (2005) suggested that symptoms of this syndrome appear in up to one-third of all cases with FTD. The age at onset is variable, with most cases being between 55-70 years of age (Hodges and Patterson 2007). The duration of the disease is also variable (from 2 to 15 years), although patients typically survive 7 to 8 years after onset (Hodges et al. 2003; Kertesz et al. 2007, 2010; Roberson et al. 2005). There is no significant difference in sex ratio, although svPPA has been sometimes more often seen in women than men (Kertesz et al. 2007).

Similarly to the nfvPPA, there is no cure for svPPA, and so the psychoeducation of family members and caregivers is of great importance. Pharmaceutical interventions have been shown to have inconsistent effects, reducing only some of the behavioral symptoms. For example, agitation and peculiar food fads may respond to treatment with selective serotonin reuptake inhibitors. In contrast, mementine does not seem to stop the progression of the cognitive decline or behavioral abnormalities associated with SVPPA (Boxer et al. 2009).

\section{Logopenic Variant PPA}

\section{Clinical Features}

Logopenic variant PPA (lvPPA), also known as LPA (Gorno-Tempini et al. 2004; Knibb et al. 2009), logopenic primary progressive aphasia (PPA-L) (Mesulam et al. 2009) or "progressive mixed aphasia" (Grossman 2010; Mesulam et al. 2008, 2009), has been only recently described as a distinct form of PPA. Due to some overlap with the nonfluent type, these patients were often in the past included under the label of nfvPPA. Although the speech output of both nfvPPA and lvPPA is slowed, with frequent word-finding pauses and phonemic paraphasias, patients with the logopenic type do not present with agrammatism, impaired motor control of speech, and aprosodia (GornoTempini et al. 2004, 2008, 2011; Henry and Gorno-Tempini 2010). In particular, patients with lvPPA do not produce telegraphic speech with missing function words and morphemes (Wilson et al. 2010). The confrontation naming of these patients is usually only moderately affected, and cases have intact general semantics and single-word comprehension, especially early in the disease course. Given that patients typically have severe difficulty repeating sentences and longer phrases, while reproduction of short, single words remaining spared, the core impairment that underlies most language deficits in IvPPA has been suggested to be a phonological short-term memory deficit (Gorno-Tempini et al. 2008). The same mechanism is said to account for defective sentence comprehension in lvPPA, which is influenced more by the length and the frequency of a sentence than its grammatical complexity.

Nonetheless, because progressive logopenia is also a feature of nfvPPA and, as the lvPPA progresses, problems with single-word comprehension and deficits with sentence-level grammar emerge. As such, patients with lvPPA might be sometimes better referred to as "progressive mixed aphasia". Apart from the defective phonological loop of working memory, subjects with lvPPA frequently have episodic memory impairment (Mesulam et al. 2008) and poor arithmetic abilities (Rohrer et al. 2010d), and so their clinical picture is often similar to that of patients with $\mathrm{AD}$. Less commonly, some individuals may also develop limb apraxia (op.cit.). Moreover, behavioral abnormalities may be present, with apathy, anxiety, irritability, and agitation being the most frequently reported features (Rohrer and Warren 2010; Rosen et al. 2006; see also Henry and GornoTempini 2010).

\section{Neuroimaging}

Analyses of cortical thinning in patients with lvPPA have revealed a pattern of damage primarily affecting the left 
posterior superior temporal and middle temporal gyri as well as the inferior parietal lobule (Gorno-Tempini et al. 2004). Less frequently, involvement of medial temporal and parietal cortex, posterior cingulated, inferior frontal cortex, contralateral temporo-parietal cortex, as well as inferior and anterior temporal regions has also been described, accompanied by white matter loss in association tracks in the left hemisphare (Gorno-Tempini et al. 2008; Migliaccio et al. 2009; Rohrer et al. 2010d). This pattern of atrophy, consistent with the clinical picture of defective function of the phonological loop, was further confirmed by left temporo-parietal hypometabolism in a fluorodeoxyglucose positron emission tomography (FDG-PET) study by Rabinovici and coworkers (2008). It is important to note that this neurodegenerative picture is very similar to that observed in AD (Frisoni et al. 2007; Migliaccio et al. 2009). Moreover, molecular imaging techniques applied to lvPPA have typically detected the presence of cortical amyloid in these subjects (Rabinovici et al. 2008). Thus, it has been recently proposed that lvPPA may be a "unihemispheric" presentation of AD (Rohrer et al. 2010e).

\section{Neuropathology}

The hypothesis that lvPPA may be suggestive of AD pathology has been confirmed not only by imaging studies showing both left temporo-parietal atrophy (Gorno-Tempini et al. 2004, 2008; Migliaccio et al. 2009) as well as amyloid deposition in these regions (Rabinovici et al. 2008), but also by higher than expected occurrence of the apolipoprotein E4 haplotype, and reduced $A \beta 42$ and elevated tau biomarkers in the cerebrospinal fluid (Rohrer et al. 2010e).

Yet, the clinical prediction of pathology in lvPPA remains difficult (Grossman 2010; Mesulam et al. 2008). For example, Grossman (2010) found that from among autopsy-confirmed cases of PPA, 24 had a diagnosis of logopenic aphasia. Although half of these cases had AD pathology, FTLD-U pathology was present in almost $40 \%$ of subjects, and the remaining few cases had FTLD-T pathology. Also, in our center, we classified patients with PPA into probable and possible groups, depending on whether non-language memory deficits were present at onset. All 10 patients with probable PPA (nfvPPA according to the new classification by Gorno-Tempini et al., 2011) showed Pick complex pathology (3 Pick's disease, 4 CBD, 3 FTD-MNDI). In contrast, patients with memory deficits showed AD pathology (Kertesz et al. 1994). The logopenic variant of PPA resembles the previous descriptions of aphasic AD (Bayles et al 1992; Appell et al. 1982), consistent with other studies of a language variant of $\mathrm{AD}$ that overlaps closely with the lvPPA syndrome (Alladi et al. 2007; Galton et al. 2000). Munoz et al. (2007b) further examined the category of possible PPA when it was uncertain whether the patient had FTD or AD, based on the presence of episodic memory difficulties and older age of onset. AD pathology was detected in all 8 cases clinically diagnosed as possible PPA. The pattern of language impairment, measured with the Western Aphasia Battery (Kertesz 2007), was more characteristic of the lvPPA than of the nfvPPA. Structural brain imaging showed mild diffuse atrophy in four patients, with focal atrophy accompanied by distinct asymmetry in the other four cases. In most instances, cerebral blood flow on hexamethylpropyleneamine oxime single photon emission computer tomography (HMPAO-SPECT) showed parietal hypoperfusion. Only one case revealed asymmetrical, left sided atrophy on postmortem. Importantly, seven of these patients also showed large argyrophilic, thorny astrocyte clusters (ATAC) in the fronto-temporo-parietal cortex and subcortical white matter. The intensely-tau immunoreactive astrocytes in ATAC were morphologically similar to the perivascular, subpial, and subependymal astrocytes in elderly brains, but ATAC differ from them by the cortical and subcortical location, widespread distribution outside the medial temporal lobe, and intense argyrophilia. These findings were confirmed by a recent study (Mesulam et al. 2008). Argyrophilic thorny astrocytes have been found in Pick disease and PSP (Yasuhara et al. 1995, Yamada et al. 1992). The location of ATAC was related to neither local variations in the load of $\mathrm{AD}$ pathology, nor the myelin density of white matter. Neither focal accentuation of AD pathology nor vascular lesions in language-related areas was observed. ATAC's were not seen in a comparison group of six cases of $\mathrm{AD}$ without a prominent aphasia syndrome. Because of the similarity of astrocytes in ATAC to those seen independently of AD pathology in Pick's disease and two reported cases of PPA, we hypothesized that ATAC's are a marker of a pathological process concurrent with $\mathrm{AD}$, and related to the focality of the clinical presentation, similar to FTD/Pick complex.

\section{Genetics}

The logopenic variant may be predominantely linked to mutations in PGRN gene. In a recent study by Rohrer and coworkers (2010d), two of 9 logopenic patients had such mutations. Interestingly, the clinical and imaging findings of these cases were mixed: in addition to posterior temporal involvement, there was damage to the left anterior temporal lobe and, expectantly, they exhibited some language features of svPPA. Moreover, another lately described case of logopenic-like progressive aphasia with a mutation in PGRN gene also had some additional features of verbal semantic association impairment as well as agrammatism and repetition deficits accompanied by inferior frontal atrophy (Rohrer et al. 2010f). Thus, these observations 


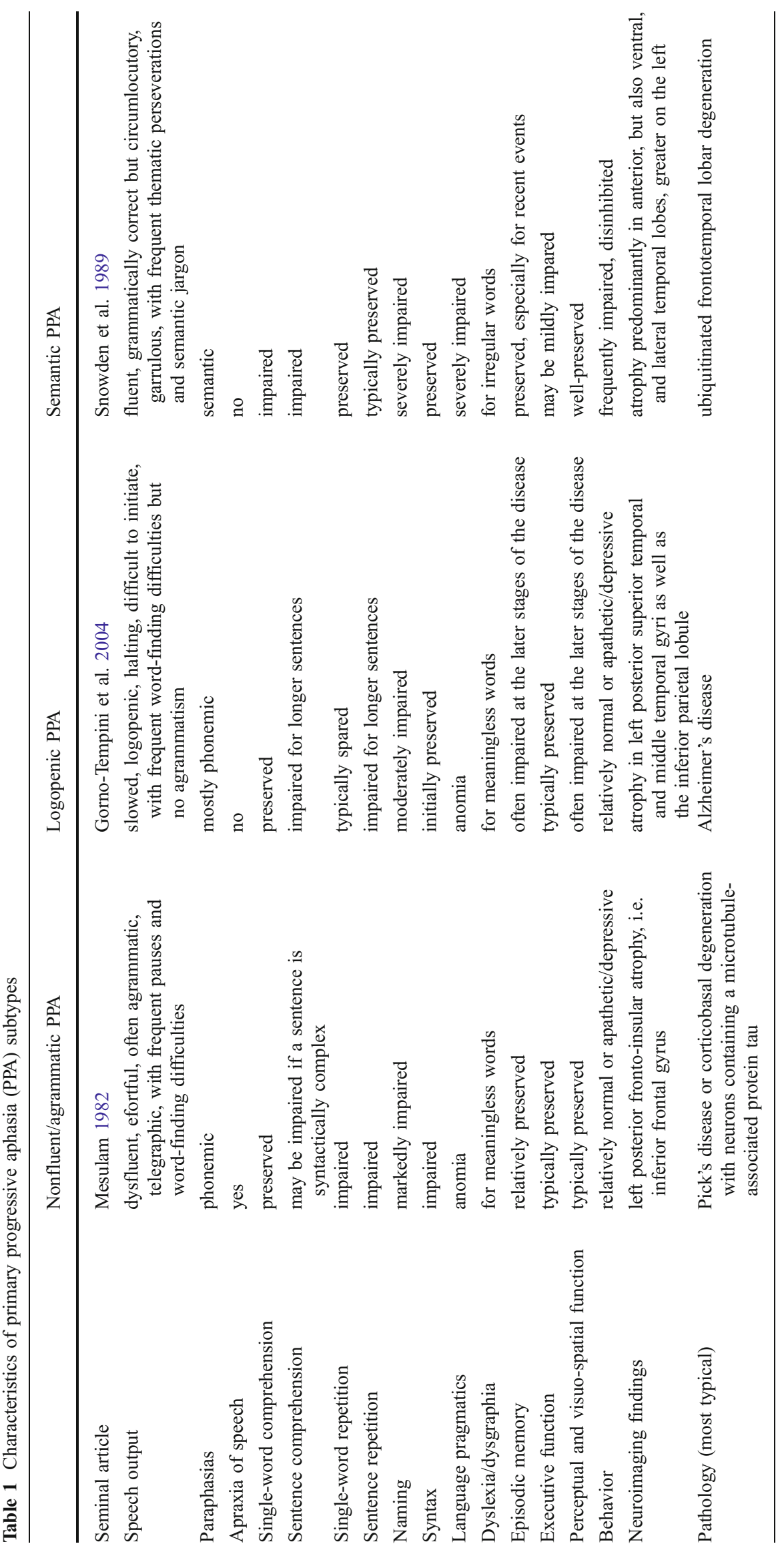


suggest that mutations in PGRN gene may cause an overlapping/mixed PPA syndrome but with a distinctive cognitive profile. This PPA syndrome would then appear to result from damage to the temporo-parietal junction as well as its functional connections in both the dorsal (involved in articulation-to-sound mapping) and ventral (involved in processing word meaning) language networks.

\section{Epidemiology and Treatment}

Because the lvPPA has been only recently described (Gorno-Tempini et al. 2004), there is still little known about its epidemiology and treatment. Nonetheless, given that most cases of lvPPA share many features with AD, the onset of its first symptoms might be similar to that in AD. Also, lvPPA may be somewhat more prevalent in woman than in man, although systematic studies are needed to test this prediction.

\section{Theoretical Implications}

Studies of patients with PPA have a number of theoretical implications that significantly contribute to our contemporary understanding of the brain-language relationship. For example, one of the key questions in neuroscience is: Which brain regions contribute to semantic memory and what role do they play? One potential answer to this query can be found in studies of patients with svPPA that typically lead to a strikingly selective multimodal impairment of semantic memory. The severity of these semantic deficits has been shown to correspond with the extent of atrophy in the anterior regions of the temporal lobes (Davies et al. 2004; Williams et al. 2005). Thus, together with the evidence that the anterior temporal lobes are widely connected to other temporal, parietal, and frontal areas (Catani and Thiebaut de Schotten 2008), but not associated with any single motor, sensory, or verbal input/output, it has been proposed that these temporal regions serve as an amodal hub with different streams of modality-specific semantic information (Hodges and Patterson 2007; Lambon Ralph et al. 2010). Although not appearing in classical models of acquired aphasia (see Geschwind 1965), the anterior temporal region may be a critical area for semantic cognition, including lexical-semantics. Since it was found that category specific semantic deficits are sometimes seen in SvPPA, it has also been proposed that some categories may be supported mainly by modality-specific feature representations (see Martin 2007). This would then explain why categories relying strongly on visually-weighted information (e.g., abstract concepts) might remain spared. Nonetheless, a number of recent studies, including research with repetitive transcranial magnetic stimulation in normal subjects, have provided strong support for the existence of a single, amodal semantic system, additionally demonstrating that most of the category specific deficits in svPPA seem to be dependent on the stimuli used, their familiarity, typicality, age of acquisition, the severity of semantic impairment, as well as the fact that some categories may have different levels of semantic content (Cipolotti and Warrington 1995; Harciarek and Kertesz 2009; Holland and Lambon Ralph 2010; Kertesz et al. 1998; Lambon Ralph et al. 2007, 2010; Pobric et al. 2010; Pulvermüller et al. 2010).

Studies of SD have also provided further support for the notion that left temporal regions are predominantly implemented in object processing, whereas the same regions in the right hemisphere are crucial for processing knowledge about people and faces (Gainotti 2007). On the other hand, evidence from the lvPPA has confirmed the critical role of the inferior parietal cortex in phonological-loop functions, and findings from nfvPPA have provided additional information regarding the role of inferior, opercular, and insular areas in speech production and syntax. Thus, these striking dissociations are likely to support the modular theory, although clinical patterns change as disease progresses, and symptoms usually begin to overlap. Such clinical convergence might, however, be explained by a progressive loss of the integrity of white matter pathways that seem to be crucial in binding cortical areas into distributed networks that mediate particular language functions (see Seeley et al. 2009).

Another important implication comes from studies by Rohrer and colleagues $(2010 \mathrm{~d}, \mathrm{f})$ who suggested that mutations in the PGRN gene might be associated with the pathological involvement of both the dorsal and ventral language pathways, with a key site of overlap in the region of the temporo-parietal junction. This would, in turn, result in an overlapping PPA syndrome with a distinctive cognitive profile. Future genetic studies are warranted, however, to

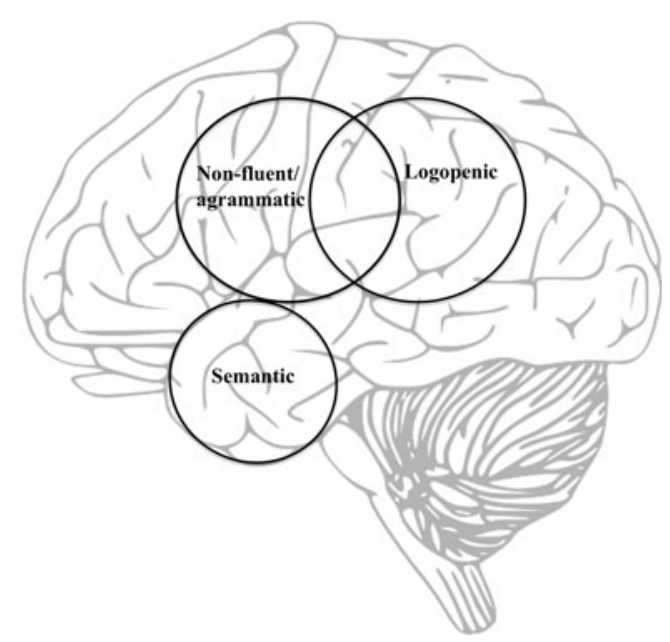

Fig. 2 A model of the neural substrates of the three primary progressive aphasia subtypes 
confirm these results as well as to better understand the complex relationship between mutations in PGRN and the development of abnormalities in the left temporo-parietal junction.

At another dimension, studies of individuals with PPA have shed some new light on the interesting association between the auditory dysfunction and impaired speech output (Goll et al. 2010). Anatomically, analysis of incoming auditory signals, speech output and monitoring of one's own voice are linked through the dorsal auditory cortical pathway(s) between frontal, parietal and posterior superior temporal cortices (Warren et al. 2006). Functionally, sensorimotor interactions mediated by this dorsal pathway have been shown to modulate spoken output in normal subjects (Wilson and Iacoboni 2006). Thus, similar to other individuals with focal damage to the posterior temporal lobe/temporo-parietal junction (Racette et al. 2006), some PPA patients may not be able to accurately transform stored templates for auditory objects into motor programs that would, in turn, result in degraded speech output. Importantly, if this hypothesis were empirically verified, it would open a new window for speech therapy in patients with different forms of aphasia.

\section{Conclusions}

Primary progressive aphasia, in the context of neurodegenerative disease such as FTD/Pick Complex or $\mathrm{AD}$, is a multifaceted, clinical condition characterized by a progressive loss of specific language functions with relative sparing of other cognitive domains (see Table 1). Based on the constellation of symptoms, PPA has been recently classified into a nonfluent, semantic, or logopenic variant. This review of the literature has primarily aimed to characterize the speech and language impairment, cognition, neuroimaging, pathology, genetics, and epidemiology associated with each of these variants. Overall, it can be posited that the nfvPPA is characterized by dysfluent and effortful speech, often combined with agrammatism. Some patients may present with predominant apraxia of speech, although they eventually develop aphasia as the disease progresses. The neuroimaging finding in nfvPPA is usually progressive atrophy within the left inferior, opercular, and insular regions, further supporting the notion of left posterior frontal areas being implemented in speech output. Pathology is most often Pick's disease or CBD (FTLD-T). Semantic variant PPA, on the other hand, is characterized by fluent, but circumlocutory speech as well as severe anomia and word-finding difficulties, all being associated with a progressive loss of lexical-semantic knowledge. As the disease progresses, the semantic impairment typically becomes multimodal and some patients may have additional difficulties processing nonverbal information (e.g., faces). The clinical picture of svPPA is often associated with bilateral atrophy of the anterior regions of the temporal lobes responsible for the multimodal integration of meaning, although in svPPA this atrophy is usually more prominent on the left side (see Fig. 2). The vast majority of these patients have FTLD-U pathology. The third and most recently described form of PPA is the logopenic variant, characterized by slowed spontaneous speech output with frequent wordfinding pauses, phonologic paraphasias, and working memory deficits. Nonetheless, in comparison to the nonfluent and the semantic variant, there are no agrammatism or single-word comprehension problems. Imaging abnormalities in lvPPA have been predominantly found in the left temporo-parietal junction area that, together with the left supplementary motor area, has been implemented in phonological integration. The pathological changes seen in lvPPA have been often those of AD.

Although some patients with PPA, especially those with semantic variant, may indeed present with a relatively clear diagnostic picture, many clinical symptoms frequenly overlap, especially as the disease progresses. Hence, determining the primary cognitive deficit in nonfluent and logopenic variant PPA remains a matter of debate. Similarly, the fluency-nonfluency distinction seems to be arbitrary because the fluency in all PPA cases is a stage-related phenomenon. Prediction of pathology also remains problematic, although a comprehensive clinical and neuropsychological assessment combined with neuroimaging seems to be most helpful. Thus, developing reliable and objective measures that allow early differential diagnosis is an important direction for future research. Additionally, more longitudinal studies of PPA are required, particularly for lvPPA, which is the least consistently defined presentation. All this would hopefully result in better understanding of the biological mechanisms underlying each PPA syndrome, and the discovery of effective treatment for this devastating condition.

Acknowledgments While preparing this manuscript, the corresponding author was receiving a "START" scholarship from the Foundation for Polish Science (FNP) as well as a scholarship from the Kosciuszko Foundation.

Open Access This article is distributed under the terms of the Creative Commons Attribution Noncommercial License which permits any noncommercial use, distribution, and reproduction in any medium, provided the original author(s) and source are credited.

\section{References}

Adlam, A. L., Patterson, K., Rogers, T. T., Nestor, P. J., Salmond, C. H., Acosta-Cabronero, J., et al. (2006). Semantic dementia and fluent primary progressive aphasia: two sides of the same coin? Brain, 129, 3066-3080. 
Alladi, S., Xuereb, J., Bak, T., Nestor, P., Knibb, J., Patterson, K., et al. (2007). Focal cortical presentations of Alzheimer's disease. Brain, 130, 2636-2645.

Alzheimer, A. (1907). Uber eine eigenartige Erkrankung der Hirnrinde. Allgemeine Zeitschrift for Psychiatrie und Psychish-Gerichtlich. Medicin, 64, 146-148.

Appell, J., Kertesz, A., \& Fisman, M. (1982). A study of language functioning in Alzheimer patients. Brain and Language, 17, 7391.

Ash, S., McMillan, C., Gunawardena, D., Avants, B., Morgan, B., Khan, A., et al. (2010). Speech errors in progressive non-fluent aphasia. Brain and Language, 113, 13-20.

Ash, S., Moore, P., Antani, S., McCawley, G., Work, M., \& Grossman, M. (2006). Trying to tell a tale: discourse impairments in progressive aphasia and frontotemporal dementia. Neurology, 66, 1405-1413.

Ash, S., Moore, P., Veseley, L., Gunawardena, D., McMillan, C., Anderson, C., et al. (2009). Non-fluent speech in frontotemporal lobar degeneration. Journal of Neurolinguistics, 22, 370-383.

Basso, A. (1988). Progressive language impairment without dementia: a case with isolated category specific semantic defect. Journal of Neurology, Neurosurgery, and Psychiatry, 51, 1201-1207.

Bayles, K. A., Tomoeda, C. K., \& Trosset, M. W. (1992). Relation of linguistic communication abilities of Alzheimer's patients to stage of disease. Brain and Language, 42, 454-472.

Benussi, L., Ghidoni, R., Pegoiani, E., Moretti, D. V., Zanetti, O., \& Binetti, G. (2009). Progranulin Leu271LeufsX10 is one of the most common FTLD and CBS associated mutations worldwide. Neurobiology of Diseases, 33, 379-385.

Bessi, V., Bagnoli, S., Nacmias, B., Tedde, A., Sorbi, S., \& Bracco, L. (2010). Semantic dementia associated with mutation V363I in the tau gene. Journal of the Neurological Sciences, 296, 112114.

Bird, H., Lambon Ralph, M. A., Patterson, K., \& Hodges, J. R. (2000). The rise and fall of frequency and imageability: noun and verb production in semantic dementia. Brain and Language, 73, $17-49$.

Blair, M., Marczinski, C. A., Davis-Faroque, N., \& Kertesz, A. (2007). A longitudinal study of language decline in Alzheimer's disease and frontotemporal dementia. Journal of the International Neuropsychological Society, 13, 237-245.

Boxer, A. L., Lipton, A. M., Womack, K., Merrilees, J., Neuhaus, J., Pavlic, D., et al. (2009). An open-label study of memantine treatment in 3 subtypes of frontotemporal lobar degeneration. Alzheimer Disease and Associated Disorders, 23, 211-217.

Bozeat, S., Gregory, C. A., Ralph, M. A., \& Hodges, J. R. (2000). Which neuropsychiatric and behavioural features distinguish frontal and temporal variants of frontotemporal dementia from Alzheimer's disease? Journal of Neurology, Neurosurgery, and Psychiatry, 69, 178-186.

Bozeat, S., Lambon Ralph, M. A., Patterson, K., Garrard, P., \& Hodges, J. R. (2000). Non-verbal semantic impairment in semantic dementia. Neuropsychologia, 38, 1207-1215.

Breedin, S., Saffran, E. M., \& Coslett, H. (1994). Reversal of the concreteness effect in a patient with semantic dementia. Cognitive Neuropsychology, 11, 617-660.

Budd, M. A., Kortte, K., Cloutman, L., Newhart, M., Gottesman, R. F., Davis, C., et al. (2010). The nature of naming errors in primary progressive aphasia versus acute post-stroke aphasia. Neuropsychology, 24, 581-589.

Caine, D., Breen, N., \& Patterson, K. (2009). Emergence and progression of 'non-semantic' deficits in semantic dementia. Cortex, 45, 483-494.

Cappa, S. F., Perani, D., Messa, C., Miozzo, A., \& Fazio, F. (1996). Varieties of progressive non-fluent aphasia. Annals of the New York Academy of Science, 777, 243-248.
Cappelletti, M., Butterworth, B., \& Kopelman, M. (2001). Spared numerical abilities in a case of semantic dementia. Neuropsychologia, 39, 1224-1239.

Caron, M. (1934). Etude clinique de la maladie Pick. Paris: Vigot.

Caselli, R. J., \& Jack, C. R., Jr. (1992). Asymmetric cortical degeneration syndromes. A proposed clinical classification. Archives of Neurology, 49, 770-780.

Catani, M., \& Thiebaut de Schotten, M. (2008). A diffusion tensor imaging tractography atlas for virtual in vivo dissections. Cortex, $44,1105-1132$.

Chan, D., Anderson, V., Pijnenburg, Y., Whitwell, J., Barnes, J., Scahill, R., et al. (2009). The clinical profile of right temporal lobe atrophy. Brain, 132, 1287-1298.

Chan, D., Fox, N. C., Scahill, R. I., Crum, W. R., Whitwell, J. L., Leschziner, G., et al. (2001). Patterns of temporal lobe atrophy in semantic dementia and Alzheimer's disease. Annals of Neurology, 49, 433-442.

Chen-Plotkin, A. S., Yuan, W., Anderson, C., McCarty Wood, E., Hurtig, H. I., Clark, C. M., et al. (2008). Corticobasal syndrome and primary progressive aphasia as manifestations of LRRK2 gene mutations. Neurology, 70, 521-527.

Chow, T. W., Hodges, J. R., Dawson, K. E., Miller, B. L., Smith, V., Mendez, M. F., et al. (2005). Referral patterns for syndromes associated with frontotemporal lobar degeneration. Alzheimer Disease and Associated Disorders, 19, 17-19.

Cipolotti, L., \& Warrington, E. K. (1995). Semantic memory and reading abilities: a case report. Journal of the International Neuropsychological Society, 1, 104-110.

Clark, D. G., Charuvastra, A., Miller, B. L., Shapira, J. S., \& Mendez, M. F. (2005). Fluent versus nonfluent primary progressive aphasia: a comparison of clinical and functional neuroimaging features. Brain and Language, 94, 54-60.

Crutch, S. J., \& Warrington, E. K. (2002). Preserved calculation skills in a case of semantic dementia. Cortex, 38, 389-399.

Daniele, A., Giustolisi, L., Silveri, M. C., Colosimo, C., \& Gainotti, G. (1994). Evidence for a possible neuroanatomical basis for lexical processing of nouns and verbs. Neuropsychologia, 32, 1325-1341.

Davies, R. R., Graham, K. S., Xuereb, J. H., Williams, G. B., \& Hodges, J. R. (2004). The human perirhinal cortex and semantic memory. The European Journal of Neuroscience, 20, 2441-2446.

Davies, R. R., Hodges, J. R., Kril, J. J., Patterson, K., Halliday, G. M., \& Xuereb, J. H. (2005). The pathological basis of semantic dementia. Brain, 128, 1984-1995.

Davies, R. R., \& Xuereb, J. H. (2007). The histopathology of frontotemporal dementia. In J. R. Hodges (Ed.), Frontotemporal dementia syndromes. New York: Cambridge University Press.

Dejerine, J., \& Serieux, P. (1897). Un cas de surdite verbale pure terminee par sensorielle suivi d'autopsie. Comptes Rendus des Seances de laSociete de Diologie, 10, 1074-1077.

Diehl, J., Grimmer, T., Drzezga, A., Riemenschneider, M., Förstl, H., \& Kurz, A. (2004). Cerebral metabolic patterns at early stages of frontotemporal dementia and semantic dementia. A PET study. Neurobiology of Aging, 25, 1051-1056.

Duffy, J. R., Peach, R. K., \& Strand, E. A. (2007). Progressive apraxia of speech as a sign of motor neuron disease. American Journal Speech-Languahe Pathology, 16, 198-208.

Edwards-Lee, T., Miller, B. L., Benson, D. F., Cummings, J. L., Russell, G. L., Boone, K., et al. (1997). The temporal variant of frontotemporal dementia. Brain, 120, 1027-1040.

Frisoni, G. B., Pievani, M., Testa, C., Sabattoli, F., Bresciani, L., Bonetti, M., et al. (2007). The topography of grey matter involvement in early and late onset Alzheimer's disease. Brain, 130, 720-730.

Fukui, T., Sugita, K., Kawamura, M., Shiota, J., \& Nakano, I. (1996). Primary progressive apraxia in Pick's disease: a clinicopathologic study. Neurology, 47, 467-473. 
Fushimi, T., Komori, K., Ikeda, M., Lambon Ralph, M. A., \& Patterson, K. (2009). The association between semantic dementia and surface dyslexia in Japanese. Neuropsychologia, 47, 10611068.

Gainotti, G. (2007). Different patterns of famous people recognition disorders in patients with right and left anterior temporal lesions: a systematic review. Neuropsychologia, 45, 1591-1607.

Galton, C. J., Patterson, K., Graham, K., Lambon-Ralph, M. A., Williams, G., Antoun, N., et al. (2001). Differing patterns of temporal atrophy in Alzheimer's disease and semantic dementia. Neurology, 57, 216-225.

Galton, C. J., Patterson, K., Xuereb, J. H., \& Hodges, J. R. (2000). Atypical and typical presentations of Alzheimer's disease: a clinical, neuropsychological, neuroimaging and pathological study of 13 cases. Brain, 123, 484-498.

Geschwind, N. (1965). Disconnexion syndromes in animals and man. I. Brain, 88, 237-294.

Gliebus, G., Bigio, E. H., Gasho, K., Mishra, M., Caplan, D., Mesulam, M. M., et al. (2010). Asymmetric TDP-43 distribution in primary progressive aphasia with progranulin mutation. Neurology, 74, 1607-1610.

Goll, J. C., Crutch, S. J., Loo, J. H., Rohrer, J. D., Frost, C., Bamiou, D. E., et al. (2010). Non-verbal sound processing in the primary progressive aphasias. Brain, 133, 272-285.

Gorno-Tempini, M. L., Brambati, S. M., Ginex, V., Ogar, J., Dronkers, N. F., Marcone, A., et al. (2008). The logopenic/phonological variant of primary progressive aphasia. Neurology, 71, 12271234.

Gorno-Tempini, M. L., Dronkers, N. F., Rankin, K. P., Ogar, J. M., Phengrasamy, L., Rosen, H. J., et al. (2004). Cognition and anatomy in three variants of primary progressive aphasia. Annals of Neurology, 55, 335-346.

Gorno-Tempini, M. L., Hillis, A. E., Weintraub, S., Kertesz, A., Mendez, M., Cappa, S. F., et al. (2011). International reccomendations for the diagnosis of primary progressive aphasia and its variants. Neurology, Feb 16. [Epub ahead of print]

Graham, N. L., Patterson, K., \& Hodges, J. R. (2004). When more yields less: speaking and writing deficits in nonfluent progressive aphasia. Neurocase, 10, 141-155.

Graham, K. S., Simons, J. S., Pratt, K. H., Patterson, K., \& Hodges, J. R. (2000). Insights from semantic dementia on the relationship between episodic and semantic memory. Neuropsychologia, 38, 313-324.

Green, H. A., \& Patterson, K. (2009). Jigsaws-a preserved ability in semantic dementia. Neuropsychologia, 47, 569-576.

Greene, J. D., Patterson, K., Xuereb, J., \& Hodges, J. R. (1996). Alzheimer disease and nonfluent progressive aphasia. Archives of Neurology, 53, 1072-1078.

Grossman, M. (2010). Primary progressive aphasia: clinicopathological correlations. Nature Review. Neurology, 6, 88-97.

Grossman, M., \& Ash, S. (2004). Primary progressive aphasia: a review. Neurocase, 10, 3-18.

Grossman, M., Mickanin, J., Onishi, K., Hughes, E., D'Esposito, M., Ding, X. S., et al. (1996). Progressive non-fluent aphasia: Language, cognitive and PET measures contrasted with probable Alzheimer's disease. Journal of Cognitive Neuroscience, 8, 135154

Gunawardena, D., Ash, S., McMillan, C., Avants, B., Gee, J., \& Grossman, M. (2010). Why are patients with progressive nonfluent aphasia nonfluent? Neurology, 75, 588-594.

Halpern, C. H., Glosser, G., Clark, R., Gee, J., Moore, P., Dennis, K., et al. (2004). Dissociation of numbers and objects in corticobasal degeneration and semantic dementia. Neurology, 62, 1163-1169.

Harciarek, M., \& Jodzio, K. (2005). Neuropsychological differences between frontotemporal dementia and Alzheimer's disease. Neuropsychology Review, 3, 131-145.
Harciarek, M., \& Kertesz, A. (2009). Longitudinal study of singleword comprehension in semantic dementia: a comparison with primary progressive aphasia and Alzheimer's disease. Aphasiology, $23,606-626$

Han, C., Kim, N. H., Kwon do, Y., Seo, W. K., \& Park, M. H. (2010). Lack of association between antisperm antibodies and language dysfunction in Alzheimer's disease. Archives of Gerontology and Geriatrics, 50, 338-340.

Henry, M. L., \& Gorno-Tempini, M. L. (2010). The logopenic variant of primary progressive aphasia. Current Opinion in Neurology, 23, 633-637.

Henschen, S. E. (1922). Klinische und Anatomische Beitrage zur Pathologie des Gehirns (Vol. 5-7). Nordiska Bokhandeln: Stockholm.

Hillis, A. E., Oh, S., \& Ken, L. (2004). Deterioration of naming nouns versus verbs in primary progressive aphasia. Annals of Neurology, $55,268-275$.

Hillis, A. E., Tuffiash, E., \& Caramazza, A. (2002). Modality-specific deterioration in naming verbs in nonfluent primary progressive aphasia. Journal of Cognitive Neuroscience, 14, 1099-1108.

Hodges, J. R., Davies, R., Xuereb, J., Kril, J., \& Halliday, G. (2003). Survival in frontotemporal dementia. Neurology, 61, 349-354.

Hodges, J. R., \& Graham, K. S. (2001). Episodic memory: insights from semantic dementia. Philosophical Transactions of the Royal Society of London, 356, 1423-1434.

Hodges, J. R., Martinos, M., Woollams, A. M., Patterson, K., \& Adlam, A. L. (2008). Repeat and Point: differentiating semantic dementia from progressive non-fluent aphasia. Cortex, 44, 12651270 .

Hodges, J. R., \& Patterson, K. (1996). Nonfluent progressive aphasia and semantic dementia: a comparative neuropsychological study. Journal of the International Neuropsychological Society, 2, 511524.

Hodges, J. R., \& Patterson, K. (2007). Semantic dementia: a unique clinicopathological syndrome. Lancet Neurology, 6, 1004-1014.

Hodges, J. R., Patterson, K., Oxbury, S., \& Funnell, E. (1992). Semantic dementia. Progressive fluent aphasia with temporal lobe atrophy. Brain, 115, 1783-806.

Hoffman, P., \& Lambon Ralph, M. A. (2011). Reverse concreteness effects are not a typical feature of semantic dementia: evidence for the hub-and-spoke model of conceptual representation. Cerebral Cortex. Feb. 3. [Epub ahead of print].

Holland, A. L., McBurney, D. H., Moossy, J., \& Reinmuth, O. M. (1985). The dissolution of language in Pick's disease with neurofibrillary tangles: a case study. Brain and Language, 24, $36-58$.

Holland, R., \& Lambon Ralph, M. A. (2010). The anterior temporal lobe semantic hub is a part of the language neural network: selective disruption of irregular past tense verbs by rTMS. Cerebral Cortex, 20, 2771-2775.

Hu, W. T., McMillan, C., Libon, D., Leight, S., Forman, M., Lee, V. M., et al. (2010). Multimodal predictors for Alzheimer disease in nonfluent primary progressive aphasia. Neurology, 75, 595-602.

Jefferies, E., Lambon Ralph, M. A., Jones, R., Bateman, D., \& Patterson, K. (2004). Surface dyslexia in semantic dementia: a comparison of the influence of consistency and regularity. Neurocase, 10, 290-299.

Jefferies, E., Patterson, K., Jones, R. W., \& Lambon Ralph, M. A. (2009). Comprehension of concrete and abstract words in semantic dementia. Neuropsychology, 23, 492-499.

Josephs, K. A., Boeve, B. F., Duffy, J. R., Smith, G. E., Knopman, D. S., Parisi, J. E., et al. (2005). Atypical progressive supranuclear palsy underlying progressive apraxia of speech and nonfluent aphasia. Neurocase, 11, 283-296.

Josephs, K. A., Duffy, J. R., Strand, E. A., Whitwell, J. L., Layton, K. F., Parisi, J. E., et al. (2006). Clinicopathological and imaging 
correlates of progressive aphasia and apraxia of speech. Brain, 129, 1385-1398.

Josephs, K. A., Whitwell, J. L., Vemuri, P., Senjem, M. L., Boeve, B. F., Knopman, D. S., et al. (2008). The anatomic correlate of prosopagnosia in semantic dementia. Neurology, 71, 16281633.

Julien, C. L., Thompson, J. C., Neary, D., \& Snowden, J. S. (2008). Arithmetic knowledge in semantic dementia: is it invariably preserved? Neuropsychologia, 46, 2732-2744.

Julien, C. L., Thompson, J. C., Neary, D., \& Snowden, J. S. (2010). Understanding quantity in semantic dementia. Cognitive Neuropsychology, 27, 3-29.

Karbe, H., Kertesz, A., \& Polk, M. (1993). Profiles of language impairment in primary progressive aphasia. Archives of Neurology, 50, 193-201.

Kashibayashi, T., Ikeda, M., Komori, K., Shinagawa, S., Shimizu, H., Toyota, Y., et al. (2010). Transition of distinctive symptoms of semantic dementia during longitudinal clinical observation. Dementia and Geriatric Cognitive Disorders, 29, 224-232.

Kertesz, A. (2006). The Banana Lady and other stories of curious behavior and speech. Victoria: Trafford.

Kertesz, A. (2007). The Western Aphasia battery-revised. New York: Grune \& Stratton.

Kertesz, A. (2008). Frontotemporal dementia: a topical review. Cognitive and Behavioral Neurology, 21, 127-133.

Kertesz, A. (2010). Anosognosia for aphasia. In G. P. Prigatano (Ed.), The study of anosognosia. New York: Oxford University Press.

Kertesz, A., Blair, M., McMonagle, P., \& Munoz, D. (2007). The diagnosis and course of frontotemporal dementia. Alzheimer Disease and Associated Disorders, 21, 155-163.

Kertesz, A., Davidson, W., \& Fox, H. (1997). Frontal behavioral inventory: diagnostic criteria for frontal lobe dementia. The Canadian Journal of Neurological Sciences, 24, 29-36.

Kertesz, A., Davidson, W., \& McCabe, P. (1998). Primary progressive semantic aphasia: a case study. Journal of the International Neuropsychological Society, 4, 388-398.

Kertesz, A., Davidson, W., McCabe, P., Takagi, K., \& Munoz, D. (2003). Primary progressive aphasia: diagnosis, varieties, evolution. Journal of the International Neuropsychological Society, 9, 710-719.

Kertesz, A., Hudson, L., Mackenzie, I. R., \& Munoz, D. G. (1994). The pathology and nosology of primary progressive aphasia. Neurology, 44, 2065-2072.

Kertesz, A., Jesso, S., Harciarek, M., Blair, M., \& McMonagle, P. (2010). What is semantic dementia?: a cohort study of diagnostic features and clinical boundaries. Archives of Neurology, 67, 483489.

Kertesz, A., Martinez-Lage, P., Davidson, W., \& Munoz, D. G. (2000). The corticobasal degeneration syndrome overlaps progressive aphasia and frontotemporal dementia. Neurology, 55, 1368-1375.

Kertesz, A., McMonagle, P., Blair, M., Davidson, W., \& Munoz, D. G. (2005). The evolution and pathology of frontotemporal dementia. Brain, 128, 1996-2005.

Kertesz, A., Morlog, D., Light, M., Blair, M., Davidson, W., Jesso, S., et al. (2008). Galantamine in frontotemporal dementia and primary progressive aphasia. Dementia and Geriatric Cognitive Disorders, 25, 178-185.

Kertesz, A., Nadkarni, N., Davidson, W., \& Thomas, A. W. (2000). The Frontal Behavioral Inventory in the differential diagnosis of frontotemporal dementia. Journal of the International Neuropsychological Society, 6, 460-468.

Knels, C., \& Danek, A. (2010). Loss of word-meaning with spared object semantics in a case of mixed primary progressive aphasia. Brain and Language, 113, 96-100.

Knibb, J. A., Woollams, A. M., Hodges, J. R., \& Patterson, K. (2009). Making sense of progressive non-fluent aphasia: an analysis of conversational speech. Brain, 132, 2734-2746.
Knibb, J. A., Xuereb, J. H., Patterson, K., \& Hodges, J. R. (2006). Clinical and pathological characterization of progressive aphasia. Annals of Neurology, 59, 156-65.

Koenig, P., Smith, E. E., \& Grossman, M. (2006). Semantic categorisation of novel objects in frontotemporal dementia. Cognitive Neuropsychology, 23, 541-562.

Lambon Ralph, M. A., Cipolotti, L., Manes, F., \& Patterson, K. (2010). Taking both sides: do unilateral anterior temporal lobe lesions disrupt semantic memory? Brain, 133, 3243-3255.

Lambon Ralph, M. A., Lowe, C., \& Rogers, T. T. (2007). Neural basis of category-specific semantic deficits for living things: evidence from semantic dementia, HSVE and a neural network model. Brain, 130, 1127-1137.

Lambon Ralph, M. A., \& Patterson, K. (2008). Generalization and differentiation in semantic memory: insights from semantic dementia. Annals of the New York Academy of Sciences, 1124, 61-76.

Lambon-Ralph, M. A., Patterson, K., Garrard, P., \& Hodges, J. R. (2003). Semantic dementia with category specificity: a comparative case-series study. Cognitive Neuropsychology, 20 (307), 326.

Lebert, F., Stekke, W., Hasenbroekx, C., \& Pasquier, F. (2004). Frontotemporal dementia: a randomised, controlled trial with trazodone. Dementia and Geriatric Cognitive Disorders, 17, $355-359$.

Libon, D. J., Xie, S. X., Moore, P., Farmer, J., Antani, S., McCawley, G., et al. (2007). Patterns of neuropsychological impairment in frontotemporal dementia. Neurology, 68, 369-375.

Libon, D. J., Xie, S. X., Wang, X., Massimo, L., Moore, P., Vesely, L., et al. (2009). Neuropsychological decline in frontotemporal lobar degeneration: a longitudinal analysis. Neuropsychology, 23, 337346.

Luzzi, S., Snowden, J. S., Neary, D., Coccia, M., Provinciali, L., \& Lambon Ralph, M. A. (2007). Distinct patterns of olfactory impairment in Alzheimer's disease, semantic dementia, frontotemporal dementia, and corticobasal degeneration. Neuropsychologia, $45,1823-1831$.

Marczinski, C. A., Davidson, W., \& Kertesz, A. (2004). A longitudinal study of behavior in frontotemporal dementia and primary progressive aphasia. Cognitive and Behavioral Neurology, 17, $185-190$.

Martin, A. (2007). The representation of object concepts in the brain. Annual Review of Psychology, 58, 25-45.

Matuszewski, V., Piolino, P., Belliard, S., de la Sayette, V., Laisney, M., Lalevée, C., et al. (2009). Patterns of autobiographical memory impairment according to disease severity in semantic dementia. Cortex, 45, 456-472.

Mayberry, E. J., Sage, K., \& Lambon Ralph, M. A. (2010). At the edge of semantic space: the breakdown of coherent concepts in semantic dementia is constrained by typicality and severity but not modality. Journal of Cognitive Neuroscience. [Epub ahead of print]

McMonagle, P., Blair, M., \& Kertesz, A. (2006). Corticobasal degeneration and progressive aphasia. Neurology, 67, 1444 1451.

Mehler, M. F., Dickson, D., Davies, P., \& Horoupian, D. S. (1986). Primary dysphasic dementia: clinical, pathological, and biochemical studies. Annals of neurology, 20, 126.

Mendez, M. F., Clark, D. G., Shapira, J. S., \& Cummings, J. L. (2003). Speech and language in progressive nonfluent aphasia compared with early Alzheimer's disease. Neurology, 61, 1108-1113.

Mesulam, M. M. (1987). Primary progressive aphasia-differentiation from Alzheimer's disease. Annals of Neurology, 22, 533-534.

Mesulam, M. M. (1982). Slowely progressive aphasia without generalized dementia. Annals of Neurology, 11, 592-598.

Mesulam, M. M. (2001). Primary progressive aphasia. Annals of Neurology, 49, 425-432. 
Mesulam, M. M. (2003). Primary progressive aphasia-a languagebased dementia. New England Journal of Medicine, 349, 15351542 .

Mesulam, M., Johnson, N., Krefft, T. A., Gass, J. M., Cannon, A. D., Adamson, J. L., et al. (2007). Progranulin mutations in primary progressive aphasia: the PPA1 and PPA3 families. Archives of Neurology, 64, 43-47.

Mesulam, M., Weineke, C., Rogalski, E., Cobia, D., Thompson, C., \& Weintraub, S. (2009). Quantitative template for subtyping primary progressive aphasia. Archives of Neurololgy, 66, 15451551.

Mesulam, M., Wicklund, A., Johnson, N., Rogalski, E., Leger, G. C., Rademaker, A., et al. (2008). Alzheimer and frontotemporal pathology in subsets of primary progressive aphasia. Annals of Neurology, 63, 709-719.

Meteyard, L., \& Patterson, K. (2009). The relation between content and structure in language production: an analysis of speech errors in semantic dementia. Brain and Language, 110, 121-134.

Migliaccio, R., Agosta, F., Rascovsky, K., Karydas, A., Bonasera, S., Rabinovici, G. D., et al. (2009). Clinical syndromes associated with posterior atrophy: early age at onset $\mathrm{AD}$ spectrum. Neurology, 73, 1571-1578.

Mondini, S., \& Semenza, C. (2006). How Berlusconi keeps his face: a neuropsychological study in a case of semantic dementia. Cortex, 42, 332-335.

Mummery, C. J., Patterson, K., Price, C. J., Ashburner, J., Frackowiak, R. S., \& Hodges, J. R. (2000). A voxel-based morphometry study of semantic dementia: relationship between temporal lobe atrophy and semantic memory. Annals of Neurology, 47, 36-45.

Munoz, D. G., Ros, R., Fatas, M., Bermejo, F., \& de Yebenes, J. G. (2007). Progressive nonfluent aphasia associated with a new mutation V363I in tau gene. American Journal of Alzheimers Disease and Other Dementias, 22, 294-299.

Munoz, D. G., Woulfe, J., \& Kertesz, A. (2007). Argyrophilic thorny astrocyte clusters in association with Alzheimer's disease pathology in possible primary progressive aphasia. Acta Neuropathologica, 114, 347-357.

Neary, D., Snowden, J. S., Gustafson, L., Passant, U., Stuss, D., Black, S., et al. (1998). Frontotemporal lobar degeneration: a consensus on clinical diagnostic criteria. Neurology, 51, 1546-1554.

Nestor, P. J., Graham, K. S., Bozeat, S., Simons, J. S., \& Hodges, J. R. (2002). Memory consolidation and the hippocampus: further evidence from studies of autobiographical memory in semantic dementia and frontal variant frontotemporal dementia. Neuropsychologia, 40, 633-654.

Nestor, P. J., Graham, N. L., Fryer, T. D., Williams, G. B., Patterson, K., \& Hodges, J. R. (2003). Progressive non-fluent aphasia is associated with hypometabolism centred on the left anterior insula. Brain, 126, 2406-2418.

Ogar, J. M., Dronkers, N. F., Brambati, S. M., Miller, B. L., \& GornoTempini, M. L. (2007). Progressive nonfluent aphasia and its characteristic motor speech deficits. Alzheimer Disease and Associated Disorders, 21, S23-30.

Patterson, K., Graham, N. L., Lambon-Ralph, M. A., \& Hodges, J. R. (2006). Progressive non-fluent aphasia is not a progressive form of non-fluent (post-stroke) aphasia. Aphasiology, 20, $1018-1034$.

Peelle, J. E., Troiani, V., Gee, J., Moore, P., McMillan, C., Vesely, L., et al. (2008). Sentence comprehension and voxel-based morphometry in progressive nonfluent aphasia, semantic dementia, and nonaphasic frontotemporal dementia. Journal of Neurolinguistics, $21,418-432$

Pick, A. (1892). Uber die Beziehung der senilen Hirnatrophie zur Aphasie. Pragischer Medizinischer Wochenschrift, 17, 165-167.

Pick, A. (1904). Uber primare progressive Demenz bei Erwachsenen. Pragischer Medizinischer Wochenschrift, 29, 417-420.
Pickering-Brown, S. M., Rollinson, S., Du Plessis, D., Morrison, K. E., Varma, A., Richardson, A. M., et al. (2008). Frequency and clinical characteristics of progranulin mutation carriers in the Manchester frontotemporal lobar degeneration cohort: comparison with patients with MAPT and no known mutations. Brain, $131,721-731$.

Pobric, G., Jefferies, E., \& Lambon Ralph, M. A. (2010). Categoryspecific versus category-general semantic impairment induced by transcranial magnetic stimulation. Current Biology, 20, 964-968.

Poeck, K., \& Luzzatti, C. (1988). Slowly progressive aphasia in three patients. The problem of accompanying neuropsychological deficit. Brain, 111, 151-168.

Pulvermüller, F., Cooper-Pye, E., Dine, C., Hauk, O., Nestor, P. J., \& Patterson, K. (2010). The word processing deficit in semantic dementia: all categories are equal, but some categories are more equal than others. Journal of Cognitive Neuroscience, 22, 2027 2041.

Rabinovici, G. D., Jagusi, W. J., Furst, A. J., Ogar, J. M., Racine, C. A., Mormino, E. C., et al. (2008). Abeta amyloid and glucose metabolism in three variants of primary progressive aphasia. Annals of Neurology, 64, 388-401.

Racette, A., Bard, C., \& Peretz, I. (2006). Making non-fluent aphasics speak: sing along! Brain, 129, 2571-2584.

Roberson, E. D., Hesse, J. H., Rose, K. D., Slama, H., Johnson, J. K., Yaffe, K., et al. (2005). Frontotemporal dementia progresses to death faster than Alzheimer disease. Neurology, 65, 719-725.

Robinson, G., \& Cipolotti, L. (2001). The selective preservation of color naming in semantic dementia. Neurocase, 7, 65-75.

Rohrer, J. D., Crutch, S. J., Warrington, E. K., \& Warren, J. D. (2010). Progranulin-associated primary progressive aphasia: a distinct phenotype? Neuropsychologia, 48, 288-297.

Rohrer, J. D., Paviour, D., Bronstein, A. M., O'Sullivan, S. S., Lees, A., \& Warren, J. D. (2010). Progressive supranuclear palsy syndrome presenting as progressive nonfluent aphasia: a neuropsychological and neuroimaging analysis. Movement Disorders, $25,179-188$.

Rohrer, J. D., Ridgway, G. R., Crutch, S. J., Hailstone, J., Goll, J. C., Clarkson, M. J., et al. (2010). Progressive logopenic/phonological aphasia: erosion of the language network. Neuroimage, 49, 984-993.

Rohrer, J. D., Rossor, M. N., \& Warren, J. D. (2010a). Syndromes of nonfluent primary progressive aphasia: a clinical and neurolinguistic analysis. Neurology, 75, 603-610.

Rohrer, J. D., Rossor, M. N., \& Warren, J. D. (2010b). Apraxia in progressive nonfluent aphasia. Journal of Neurology, 257, 569574.

Rohrer, J. D., Rossor, M. N., \& Warren, J. D. (2010e). Alzheimer's pathology in primary progressive aphasia. Neurobiology of Aging. [Epub ahead of print].

Rohrer, J. D., \& Warren, J. D. (2010). Phenomenology and anatomy of abnormal behaviours in primary progressive aphasia. Journal of the Neurological Sciiences, 29, 35-8.

Rohrer, J. D., Warren, J. D., Barnes, J., Mead, S., Beck, J., Pepple, T., et al. (2008). Mapping the progression of progranulin-associated frontotemporal lobar degeneration. Nature Clinical Practice. Neurology, 4, 455-460.

Rosen, H. J., Gorno-Tempini, M. L., Goldman, W. P., Perry, R. J., Schuff, N., Weiner, M., et al. (2002). Patterns of brain atrophy in frontotemporal dementia and semantic dementia. Neurology, 58, 198-208.

Rosen, H. J., Allison, S. C., Ogar, J. M., Amici, S., Rose, K., Dronkers, N., et al. (2006). Behavioral features in semantic dementia vs other forms of progressive aphasias. Neurology, 67, $1752-1756$.

Rosenfeld, M. (1909). Die partielle Gorsshirnatrophie. Journal fur Psychologie und Neurologie, 14, 115-130. 
Scahill, V. L., Hodges, J. R., \& Graham, K. S. (2005). Can episodic memory tasks differentiate semantic dementia from Alzheimer's disease? Neurocase, 11, 441-451.

Schwartz, M. F., Marin, O. S., \& Saffran, E. M. (1979). Dissociations of language function in dementia: a case study. Brain and Language, 7, 277-306.

Seeley, W. W., Bauer, A. M., Miller, B. L., Gorno-Tempini, M. L., Kramer, J. H., Weiner, M., et al. (2005). The natural history of temporal variant frontotemporal dementia. Neurology, 64, 1384-1390.

Seeley, W. W., Crawford, R. K., Zhou, J., Miller, B. L., \& Greicius, M. D. (2009). Neurodegenerative diseases target large-scale human brain networks. Neuron, 62, 42-52.

Selnes, O. A., \& Harciarek, M. (2006). Primary progressive aphasia without dementia. [In:] K. Brown (ed.), The encyclopedia of language and linguistics-Second Edition, Elsevier Science.

Serieux, P. (1893). Sur un cas de surdite verbale pure. Revue de Medecin, 13, 733-750.

Simons, J. S., Graham, K. S., Galton, C. J., Patterson, K., \& Hodges, J. R. (2001). Semantic knowledge and episodic memory for faces in semantic dementia. Neuropsychology, 15, 101-114.

Snowden, J. S., Bathgate, D., Varma, A., Blackshaw, A., Gibbons, Z. C., \& Neary, D. (2001). Distinct behavioural profiles in frontotemporal dementia and semantic dementia. Journal of Neurology, Neurosurgery, and Psychiatry, 70, 323-332.

Snowden, J. S., Goulding, P. J., \& Neary, D. (1989). Semantic dementia: a form of circumscribed cerebral atrophy. Behavioural Neurology, 2, 167-182.

Snowden, J., Neary, D., \& Mann, D. (2007). Frontotemporal lobar degeneration: clinical and pathological relationships. Acta Neuropathologica, 114, 31-38.

Snowden, J. S., Pickering-Brown, S. M., Mackenzie, I. R., Richardson, A. M., Varma, A., Neary, D., et al. (2006). Progranulin gene mutations associated with frontotemporal dementia and progressive non-fluent aphasia. Brain, 129, 3091-3102.

Soliveri, P., Piacentini, S., Carella, F., Testa, D., Ciano, C., \& Girotti, F. (2003). Progressive dysarthria: definition and clinical follow-up. Neurological Sciences, 24, 211-212.

Sonty, S. P., Mesulam, M. M., Weintraub, S., Johnson, N. A., Parrish, T. B., \& Gitelman, D. R. (2007). Altered effective connectivity within the language network in primary progressive aphasia. Journal of Neuroscience, 27, 1334-1345.

Swartz, J. R., Miller, B. L., Lesser, I. M., \& Darby, A. L. (1997). Frontotemporal dementia: treatment response to serotonin selective reuptake inhibitors. Journal of Clinical Psychiatry, 58, 212-216.

Tanabe, H., Ikeda, M., Nakagawa, Y., Yamamoto, H., Ikejiri, Y., \& Kazui, H. (1992). Gogi (worn meaning) aphasia and semantic memory for words. Higher Brain in Functional Research, 12, 153-167.

Thompson, S. A., Patterson, K., \& Hodges, J. R. (2003). Left/right asymmetry of atrophy in semantic dementia: behavioralcognitive implications. Neurology, 61, 1196-1203.

Turner, R. S., Kenyon, L. C., Trojanowski, J. Q., Gonatas, N., \& Grossman, M. (1996). Clinical, neuroimaging, and pathologic features of progressive nonfluent aphasia. Annals of Neurology, $39,166-173$.

Warren, J. E., Wise, R. J., \& Warren, J. D. (2006). Sounds do-able: auditory-motor transformations and the posterior temporal plane. Trends in Neurosciences, 28, 636-643.
Warrington, E. K. (1975). The selective impairment of semantic memory. Quarterly Journal of Experimental Psychology, 27, 635-657.

Wechsler, A. (1977). Presenile dementia presenting as aphasia. Journal of Neurology, Neurosurgery, and Psychiatry, 40, 303-305.

Weinstein, J., Koenig, P., Gunawardena, D., McMillan, C., Bonner, M., \& Grossman, M. (2011). Preserved musical semantic memory in semantic dementia. Archives of Neurology, 68, 248-250.

Weintraub, S., Fahey, C., Johnson, N., Mesulam, M. M., Gitelman, D. R., Weitner, B. B., et al. (2006). Vasectomy in men with primary progressive aphasia. Cognitive and Behavioral Neurology, 19, 190-193.

Weintraub, S., Mesulam, M. M., Wieneke, C., Rademaker, A., Rogalski, E. J., \& Thompson, C. K. (2009). The northwestern anagram test: measuring sentence production in primary progressive aphasia. American Journal of Alzheimers Disease and Other Dementias, 24, 408-416.

Weintraub, S., Rubin, N. P., \& Mesulam, M. M. (1990). Primary progressive aphasia. Longitudinal course, neuropsychological profile, and language features. Archives of Neurology, 47, 13291335 .

Westbury, C., \& Bub, D. (1997). Primary progressive aphasia: a review of 112 cases. Brain and Language, 60, 381-406.

Whitwell, J. L., Avula, R., Senjem, M. L., Kantarci, K., Weigand, S. D., Samikoglu, A., et al. (2010). Gray and white matter water diffusion in the syndromic variants of frontotemporal dementia. Neurology, 74, 1279-1287.

Williams, G. B., Nestor, P. J., \& Hodges, J. R. (2005). Neural correlates of semantic and behavioural deficits in frontotemporal dementia. Neuroimage, 24, 1042-1051.

Wilson, S. M., Brambati, S. M., Henry, R. G., Handwerker, D. A., Agosta, F., Miller, B. L., et al. (2009). The neural basis of surface dyslexia in semantic dementia. Brain, 132, 71-86.

Wilson, S. M., Henry, M. L., Besbris, M., Ogar, J. M., Dronkers, N. F., Jarrold, W., et al. (2010). Connected speech production in three variants of primary progressive aphasia. Brain, 133, 2069-2088.

Wilson, S. M., \& Iacoboni, M. (2006). Neural responses to non-native phonemes varying in producibility: evidence for the sensorimotor nature of speech perception. Neuroimage, 33, 316-325.

Wilson, S. M., Ogar, J. M., Laluz, V., Growdon, M., Jang, J., Glenn, S., et al. (2009). Automated MRI-based classification of primary progressive aphasia variants. Neuroimage, 47, 1558-1567.

Yamada, T., McGeer, P. L., \& McGeer, E. G. (1992). Appearance of paired nucleated, Tau-positive glia in patients with progressive supranuclear palsy brain tissue. Neuroscience Letters, 135, 99102.

Yasuhara, O., Matsuo, A., Tooyama, I., Kimura, H., McGeer, E. G., \& McGeer, P. L. (1995). Pick's disease immunohistochemistry: new alterations and Alzheimer's disease comparisons. Acta Neuropathologica (Berlin), 89, 322-330.

Yi, H. A., Moore, P., \& Grossman, M. (2007). Reversal of the concreteness effect for verbs in patients with semantic dementia. Neuropsychology, 21, 9-19.

Zamarian, L., Karner, E., Benke, T., Donnemiller, E., \& Delazer, M. (2006). Knowing $7 \times 8$, but not the meaning of 'elephant': evidence for the dissociation between numerical and non-numerical semantic knowledge. Neuropsychologia, 44, 1708-1723. 